\title{
Sustaining the Gains and Addressing the Threats and Challenges for the Continuum from Malaria Control to Elimination
}

\author{
Ambrose O. Talisuna ${ }^{1,2^{*}}$ \\ ${ }^{1}$ Department of Public Health Research, KEMRI-Welcome Trust Research Program, Kenya \\ ${ }^{2}$ Centre for Tropical Medicine, Nuffield Department of Clinical Medicine, University of Oxford, University of Oxford-KEMRI-Wellcome Trust Research Programme, Nairobi, \\ Kenya
}

In 2007, Bill and Melinda Gates, at a malaria forum in Seattle, USA, literally shocked the global malaria community when they made a clarion call to eliminate (reduce incidence to zero in a given geographical location) and ultimately eradicate malaria (reduce incidence to zero globally) [1]. This clarion call was followed up by the World Health Organization (WHO) and the Roll Back Malaria Partnership (RBM) through the development of the global malaria action plan (GMAP) 2008-2015, with a critical message to Scale Up For Impact (SUFI) [2]. Thanks to major funding bodies such as the Global Fund to fight Aids, Tuberculosis and Malaria, the United States Presidents Malaria Initiative (PMI), the Bill and Melinda Gates foundation and several bilateral and multi-lateral donors, the scale-up of malaria interventions during the last decade has contributed to a broad shift in malaria epidemiology and transmission intensity globally. Several countries have seen big reductions in malaria, but there are also others where the disease remains stubbornly high [3]. The changing epidemiology of malaria has resulted in diverse ambitions with some countries aiming at elimination, yet for others malaria elimination is currently not technically, operationally or financially feasible as they must first sustain the path of scaling up for impact in order to reduce the disease burden.

In May 2015, the 68th World Health Assembly (WHA) held in Geneva endorsed the Global Malaria Technical Strategy (GTS), 2016-2030 [4]. The GTS is probably the most bold since the eighth WHA in 1955 endorsed the Global Malaria Eradication Programme $[5,6]$. To the credit of the WHO global malaria programme (GMP), the GTS (2016-2030), was developed through an inclusive and highly participatory process under the guidance of a steering committee composed of leading malaria technical experts, scientists and country representatives with oversight provided by the Malaria Policy Advisory Committee (MPAC) [4]. During the GTS development process, the WHO consulted all affected countries through a series of 7 regional consultations and in July-August 2014, held a public web consultation, during which members of the global health community, including nongovernmental organizations in official relations with WHO, had an opportunity to comment on the draft GTS. The GTS has five strategic principles namely:

1. All countries can accelerate efforts towards elimination through combinations of interventions tailored to local contexts.

2. Country ownership and leadership, with involvement and participation of communities, are essential to accelerating progress through a multisectoral approach.

3. Improved surveillance, monitoring and evaluation, as well as stratification by malaria disease burden, are required to optimize the implementation of malaria interventions.

4. Equity in access to services especially for the most vulnerable and hard-to-reach populations is essential.

5. Innovation in tools and implementation approaches will enable countries to maximize their progression along the path to elimination.

The GTS also articulates the strategic framework pillars and supporting elements to maximize impact of today's life-saving tools:

Pillar 1. Ensure universal access to malaria prevention, diagnosis and treatment.

Pillar 2. Accelerate efforts towards elimination and attainment of malaria-free status.

Pillar 3. Transform malaria surveillance into a core intervention.

There are two supporting elements:

Supporting element 1 is harnessing innovation and expanding research: Basic research to foster innovation and the development of new and improved tools; implementation research to optimize impact and cost-effectiveness of existing tools and strategies and action to facilitate rapid uptake of new tools, interventions and strategies.

Supporting element 2 is strengthening the enabling environment which should involve: Strong political and financial commitments; Multisectoral approaches, and cross-border and regional collaborations; Stewardship of the entire heath system including the private sector, with strong regulatory support and capacity development for both effective programme management and research. The bold vision is a world free of malaria and the four goals are: 1) to reduce malaria mortality rates globally compared with 2015 , by at least $40 \%$ by 2020 , at least $75 \%$ by 2025 and at least $90 \%$ by $2030 ; 2$ ) to reduce malaria case incidence globally compared with 2015 , by at least $40 \%$ by 2020 , at least $75 \%$ by 2025 and at least $90 \%$ by $2030 ; 3$ ) to eliminate malaria from countries in which malaria was transmitted in 2015, at least 10 countries by 2020,20 countries by 2025 , and at least 35 countries by 2030; and 4) to eliminate malaria from countries in which malaria was transmitted in 2015 through prevention of re-establishment [4]. The estimated costs of implementation of the GTS are projected to be US\$ 6.4 billion per year by 2020 , US $\$ 7.7$ billion per year by 2025 , US $\$ 8.7$ billion per year by 2030 or a total of US $\$ 102.3$ billion for 2016-2030 but this will be reviewed regularly. An additional $\$ 0.7$ billion per year will be required for research and development (R\&D) [4].

*Corresponding author: Ambrose O Talisuna, Public Health Research Department, University of Oxford-KEMRI-Wellcome Trust Research Programme, P.O Box 43640, 00100, Nairobi, Kenya, Tel: +254 202710672; E-mail: atalisuna@ kemri-wellcome.org

Received June 16, 2015; Accepted June 17, 2015; Published June 24, 2015

Citation: Talisuna AO (2015) Sustaining the Gains and Addressing the Threats and Challenges for the Continuum from Malaria Control to Elimination. Malar Cont Elimination 4: e128. doi:10.4172/2470-6965.1000e128

Copyright: ( 2015 Talisuna AO . This is an open-access article distributed under the terms of the Creative Commons Attribution License, which permits unrestricted use, distribution, and reproduction in any medium, provided the original author and source are credited. 
The GTS and the current developments in malaria control/ elimination are encouraging. However, there are major threats and challenges to the continuum from control to elimination. Firstly, as the malaria epidemiology changes, there will be a need for malaria endemic countries irrespective of their current malaria control/elimination context and ambitions to improve their malaria programming. A "one size fits all" technical approach for malaria control is no longer the most efficient use of funding. While such as an approach was understandable to address the malaria "epidemic" during the late 1990 s, it is likely not sustainable with a heterogeneity of malaria transmission even at country level. Secondly, there is a need to sustain funding. Of particular concern is the limited domestic financing from malaria endemic countries, especially in Africa. This sad scenario has to urgently change because malaria endemic countries need to take ownership of their programmes through strong political and financial support. Thirdly, as the threats such as drug and insecticide resistance become real $[7,8]$, there will be a need to commit more resources to research and development (R\&D) for new tools such as vaccines, new medicines, new vector control tools and new diagnosis tools. Fourthly, as countries move from control to elimination, there will be a need for robust surveillance systems to be able to measure changes and also to help target interventions to the epidemiological context. This will require investments in training epidemiologists, entomologists, programme management staff, geographical information system specialists and procurement and supply chain specialists. With the changing epidemiological context it is vital both to maximize value for money by more intelligent targeting to justify the health and economic benefits of sustaining control/elimination so that financial support is maintained.

Finally, making country-level malaria policy, practice and resource allocation based more on evidence will require a transformational shift in the culture of decision-making. There will be a need to identify evidence gaps, analyze and use information for strategic planning and stronger systems for systematically collecting high quality data. Malaria risk mapping and stratification should become a corner stone to help targeting different epidemiological contexts with appropriate and technically sound malaria control strategies. Data on malaria transmission should also guide introduction of new strategies relevant to malaria epidemiology and transmission intensity. Additional layers of information on the mosquito, parasite and people's behavior, intervention coverage and quality of health services should be routinely used to define national malaria strategies and guide cost-effective allocation of health sector resources.

\section{References}

1. Melinda French Gates (2007) Bill and Melinda Gates Foundation Malaria Forum.

2. The Roll Back Malaria Partnership (2011).World Health Organization. 20 avenue Appia 1211 Geneva 27, Switzerland.

3. WHO (2014) World Malaria Report. World Health Organization. 20 Avenue Appia, 1211 Geneva 27, Switzerland.

4. WHO (2015) Global technical strategy for malaria (2016-2030). World Health Organization. 20 Avenue Appia, 1211 Geneva 27, Switzerland.

5. WHO (1955) Eighth World Health Assembly (Mexico, D.F., 10-27 May 1955) Official records of the World Health Organization. Nu 63. Geneva. pp 236-240.

6. Nájera AJ, González-Silva M, Pedro L, Alonso PL. (2011) Some Lessons for the Future from the Global Malaria Eradication Programme (1955-1969). PLoS Med 8: e1000412.

7. WHO (2010) Global plan for Artemisinin Resistance Containment (GPARC) World Health Organization. 20 Avenue Appia, 1211 Geneva 27, Switzerland.

8. WHO (2012) Global plan for insecticide resistance management in malaria vectors (GPIRM). World Health Organization. 20 Avenue Appia, 1211 Geneva 